\title{
TRP Receptors in Arthritis, Gaining Knowledge for Translation from Experimental Models
}

\author{
E.S. Fernandes ${ }^{1,2}$, S. Awal ${ }^{2}$, R. Karadaghi ${ }^{2}$ and S.D. Brain ${ }^{2, *}$ \\ ${ }^{\text {I} P r o g r a m a ~ d e ~ P o ́ s-G r a d u a c ̧ a ̃ o ~ e m, ~ B i o l o g i a ~ P a r a s i t a ́ r i a, ~ U n i v e r s i d a d e ~ C e u m a, ~ M A, ~ B r a z i l ; ~}{ }^{2}$ Centre of Integrative \\ Biomedicine and BHF Centre for Cardiovascular Excellence, King's College London, London Se1 9NH, UK
}

\begin{abstract}
Arthritis is a condition characterised by mainly pain, reduced joint movement and signs of inflammation, such as swelling. The disorder has many different types, of which osteoarthritis (a degenerative joint disease) and rheumatoid arthritis (a chronic autoimmune disease) are the two most common forms. There are $>6$ million sufferers in the UK and both conditions have a huge potential to impair capabilities and contribute to social and economic burdens. Whilst there are a wide range of arthritic therapies available, many patients under treatment complain of poor pain relief. Thus there is a need for novel therapeutic approaches, and the transient receptor potential (TRP) family of receptor channels has been investigated. One particular area of recent research has been the ligand-gated transient receptor potential vanilloid 1 (TRPV1) channel. Findings from numerous pre-clinical models and scientific studies have shown that TRPV1 desensitisation, or the use of TRPV1 antagonists alleviates pain and some inflammatory aspects. New findings have started to unveil the potential of other TRP channels in mediating arthritic pain and inflammation. With the understanding that the currently available treatments for arthritis are limited, researchers have looked into the exciting prospect that TRP receptor antagonists may be developed into effective, specific drugs, which would potentially protect against the complications of arthritis. These antagonists are still under development, although only data from studies from pre-clinical models are currently available. This review acts to summarize knowledge of the potential influence of TRP receptors in arthritis to date.
\end{abstract}

Keywords: TRP receptors, arthritis, knowledge.

\section{INTRODUCTION}

\subsection{Arthritis}

Arthritis is defined as the inflammation of one (or more) joint(s), and characterised by pain, swelling, warmth, redness (of the overlying skin) and a diminished range of joint movements. Considering the widespread occurrence of arthritis, it is not surprising that there is much research interest. There are many different types of arthritis, but the most common are rheumatoid arthritis (RA) and osteoarthritis (OA), of which osteo-arthritis affects most, especially the elderly.

RA affects people of different ages; it is most common in women and is characterized by a marked inflammation of the synovium. Although the inflammatory changes associated with RA have been extensively investigated, their actual trigger remains unclear. RA is classified based on the presence of joint stiffness following a prolonged rest, rheumatoid factors (RF or RhF), auto-antibodies [1] and increased synovium/synovial fluid buildup in the joint, along with bone erosion [2] Fig. (1). OA is a highly common degenerative joint disease that isoften painful and directly associated with ageing and associated previous injuries, such as from certain sports and occupations [3]. By comparison to

\footnotetext{
*Address correspondence to this author at the Centre of Integrative Biomedicine and BHF Centre for Cardiovascular Excellence, King's College London, London Se1 9NH UK; Tel: 0044848 4453;

E-mail: sue.brain@kcl.ac.uk
}

RA it is considered to have a less overt inflammatory component, although a relatively mild synovial inflammation is present in OA. Its diagnosis relies on damaged bone surface with the formation of osteophytes, reduced mobility of the joint and pain associated to movement [4].

With the advances and efforts to comprehend the mechanisms underlying both RA and $\mathrm{OA}$, there are a range of local and systemic treatments available most targeting RA, especially its related pain and the progression of the immune response. The most common treatments are the nonsteroidal anti-inflammatory drugs (NSAIDs) that relieve pain and in some cases inflammation, but do not halt the ongoing disease process. There are side-effects associated with many of the treatments and this is one of the reasons that combination drug treatments have been developed; in the hope that the use of lower doses of each drug, when taken in combination, lessens side effects. Recent clinical research and therapeutic approaches have concentrated mainly on targeting rheumatoid arthritis in an aggressive manner at an early time point, through use of disease modifying antirheumatic drugs (DMARDS), often in combination with each other or with NSAIDs [5]. Furthermore, the 'biologics' are being increasingly used that provide treatment in the form of antibodies against cytokines or other proinflammatory component. These are good when effective, with relatively few side effects, but are associated with large expense which limits access. The use of biologics such as anti-TNF drugs to treat RA has improved the life quality of patients, especially when DMARDs and classic anti- 
inflammatory drugs are ineffective. Their use in combination with established DMARDS is also being developed. On the other hand, there are relatively few treatments available for the treatment of OA [6].

Overall there is an unmet need and it is understood that research into new solutions for the management of RA and $\mathrm{OA}$ are essential. This review will discuss the relevance of transient receptor potential (TRP) channel-dependent mechanisms in both the establishment and the maintenance of RA and OA and will also address the potential of these channels as pharmacological targets for the treatment of arthritis. We will first examine the fundamental studies into the role of capsaicin that act via the TRP vanilloid 1 (TRPV1) receptor to deplete and desensitise the sensory nerves, followed by pre-clinical studies involving TRPV1 knockout mice, and more latterly selective TRPV1 antagonists. Finally we will review the fragmented evidence that other TRP receptors, especially TRPA1, may have an important role in arthritis. The major studies involving these two TRP channels in arthritis are summarized in the Table 1.

\section{CAPSAICIN}

\subsection{The History of Capsaicin and Evidence from Rodent Models of Arthritis}

The pungent substance of the capsicum was initially extracted and named as capsaicin in 1846 by Thresh [31] with its chemical structure determined nearly a century later [32]. Capsaicin administration evokes the classical signs of inflammation which are associated to neuronal sensitization and thus pain; known as neurogenic inflammation. The existence of a capsaicin receptor was initially postulated by Szolcsànyi and Jancsó-Gábor in 1975 [33]. Early researchers in 1974-1977 gathered evidence from electrophysiological recordings that capsaicin sensitive afferents were in fact shown to be c-polymodal nociceptors [34]. However, a more comprehensive analysis of the interaction of capsaicin and its receptor was conveyed following the molecular identification of the structure of the receptor [7,35].

It was later established that capsaicin exerts its effects by activating TRPV1 and in turn, causes the release of neuropeptides such as substance P (SP) and calcitoninrelated peptide (CGRP) from sensory neurons which accounts for the many of the vasoactive and inflammatory effects of this compound (see [36]; Fig. (1).

Indeed, reports in the 1980s showed that SP levels were elevated in the sciatic nerve of rats with severe arthritis [37]. This was later confirmed with increased levels of SP observed in the sciatic nerve and in the knee joint capsule [38]. These findings suggest that SP released from the peripheral nerve endings and the primary afferents stimulating the area is associated with joint dysfunction in animal models of arthritis [37]. SP was soon shown to contribute to the initiation and the maintenance of joint inflammation [39]. Indeed, over the last several decades there has been an understanding that substance $\mathrm{P}$ plays a role in arthritis. $\mathrm{NK}_{1}$ receptors for substance $\mathrm{P}$ are located within components of the joints, i.e. on primary afferents and also on immune cells [40]. Moreover, the effect of $\mathrm{NK}_{1}$ receptor antagonists was shown to be effective in alleviating arthritis in animal models $[41,42]$. However, several attempts have been made to create clinically effective tachykinin $\mathrm{NK}_{1}$ receptor antagonists to attenuate arthritic symptoms. Unfortunately, these studies did not translate into new treatments for arthritis (e.g. [43]). Indeed the clinical trials with $\mathrm{NK}_{1}$ antagonists did not show any benefit in arthritis or in other types of inflammatory pain conditions. The lack of improvement of human arthritis by $\mathrm{NK}_{1}$ antagonists is still of debate with respect to the specific roles of central and peripheral substance $\mathrm{P}$ release in arthritis and also in relation to the potency of the drugs tested in the clinical trials [44, 45]. Similarly, CGRP has been implicated in the inflammatory response and pain associated to arthritis, contributing to the angiogenesis and increased neuronal growth in the arthritic joint [46-49]. The involvement of these neuropeptides in arthritis remains under investigation. For example, recent research has shown that biologics such as etanercept, which is a dimer formation of the soluble TNF $\alpha$ receptor, have the ability to reduce levels of substance $\mathrm{P}$ and CGRP as the arthritis improves [50].

An important property of capsaicin is that in addition to causing pain, its repeated administration can cause analgesia [51-53]. This effect, called neuronal desensitisation, also accounts for the reduction of the inflammatory pathways mediated by sensory neuron activation. Indeed, capsaicin is suggested to be unique in that once it activates a channel; it results in the TRPV1 channel undergoing a refractory period of desensitisation, where it becomes unresponsive to many stimuli, including capsaicin [54]. More recently it has been suggested that this may be more appropriately called 'defunctionalisation' as the animal or the area of the human treated becomes refractory to general sensory nerve stimulation and this is now acknowledged to be important in the ability of capsaicin treatment to cause pain relief [55, 56].

Despite the historical knowledge that capsaicin administration can alleviate pain and reduce inflammation, it was not too long ago that this compound was first used to treat the symptoms of arthritic pain as discussed in the next section. Following neuronal desensitisation by capsaicin, the activation of the neuronal pathways, including SP and CGRP release, becomes impaired as also does the activation of pathways depending on these neuropeptides. In 1983, Colpaert and collaborators [37] showed that the inflammation observed in adjuvant-induced arthritis was alleviated in rats pre-treated repeatedly with capsaicin. In addition, neonatal treatment with capsaicin was shown to reduce both thermal and mechanical hyperalgesia in rats with adjuvant-induced arthritis [57]. The same study also showed that capsaicin pre-treatment attenuated the weight loss associated with arthritis and improved locomotion. Possibly, low doses $(0.075 \%)$ of capsaicin induce only a slow release and consequent depletion of pro-inflammatory neuropeptides (SP and CGRP). This may allow the release of antiinflammatory peptides such as somatostatin or opioid peptides in a functional, relevant manner. Indeed, in a Freund's adjuvant-induced arthritis model of arthritis, somatostatin was shown to be released from the capsaicinsensitive nerve endings and exert a systemic antiinflammatory action [58]. 
Table 1. Major Studies Involving TRPV1 and TRPA1 in Arthritis

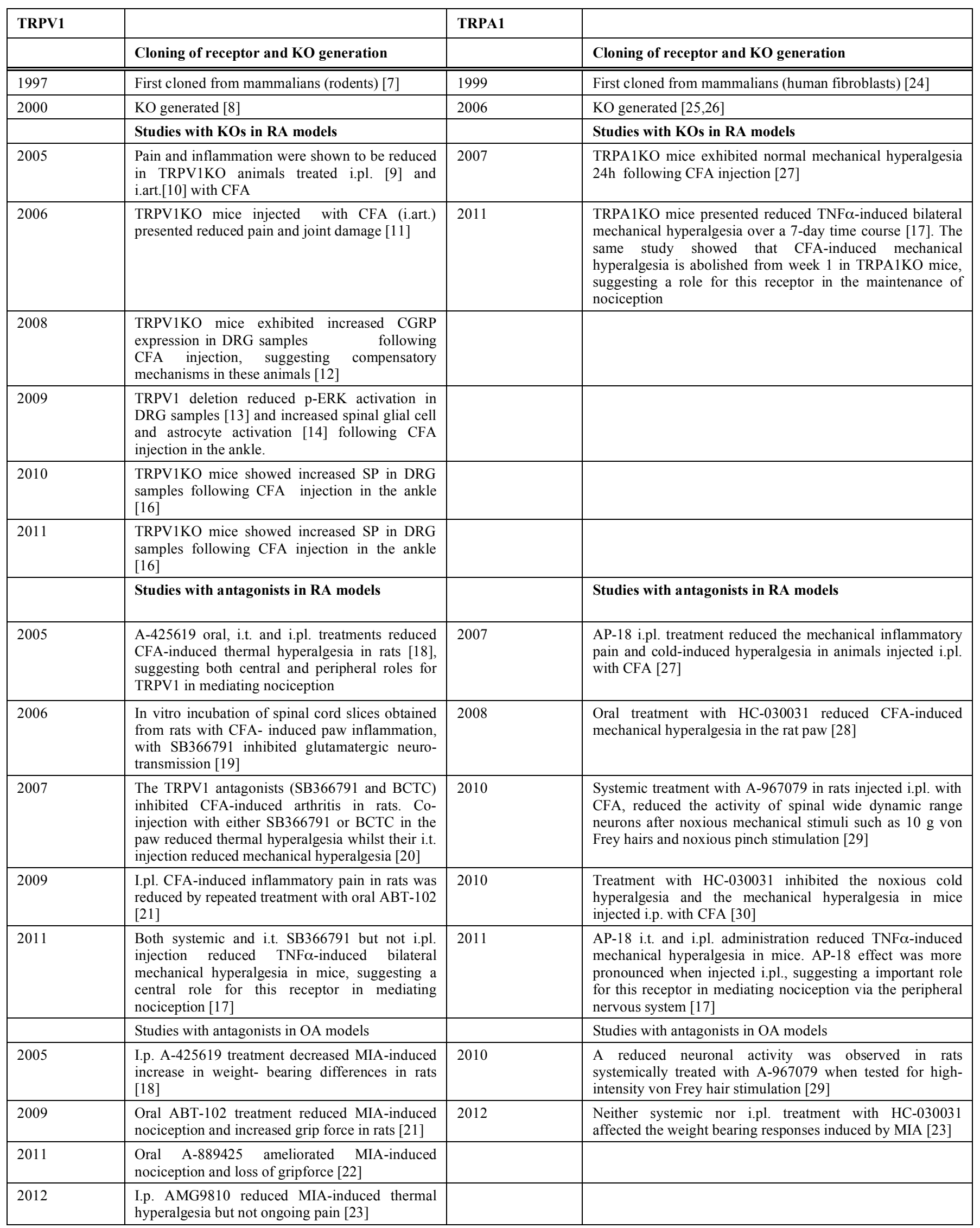




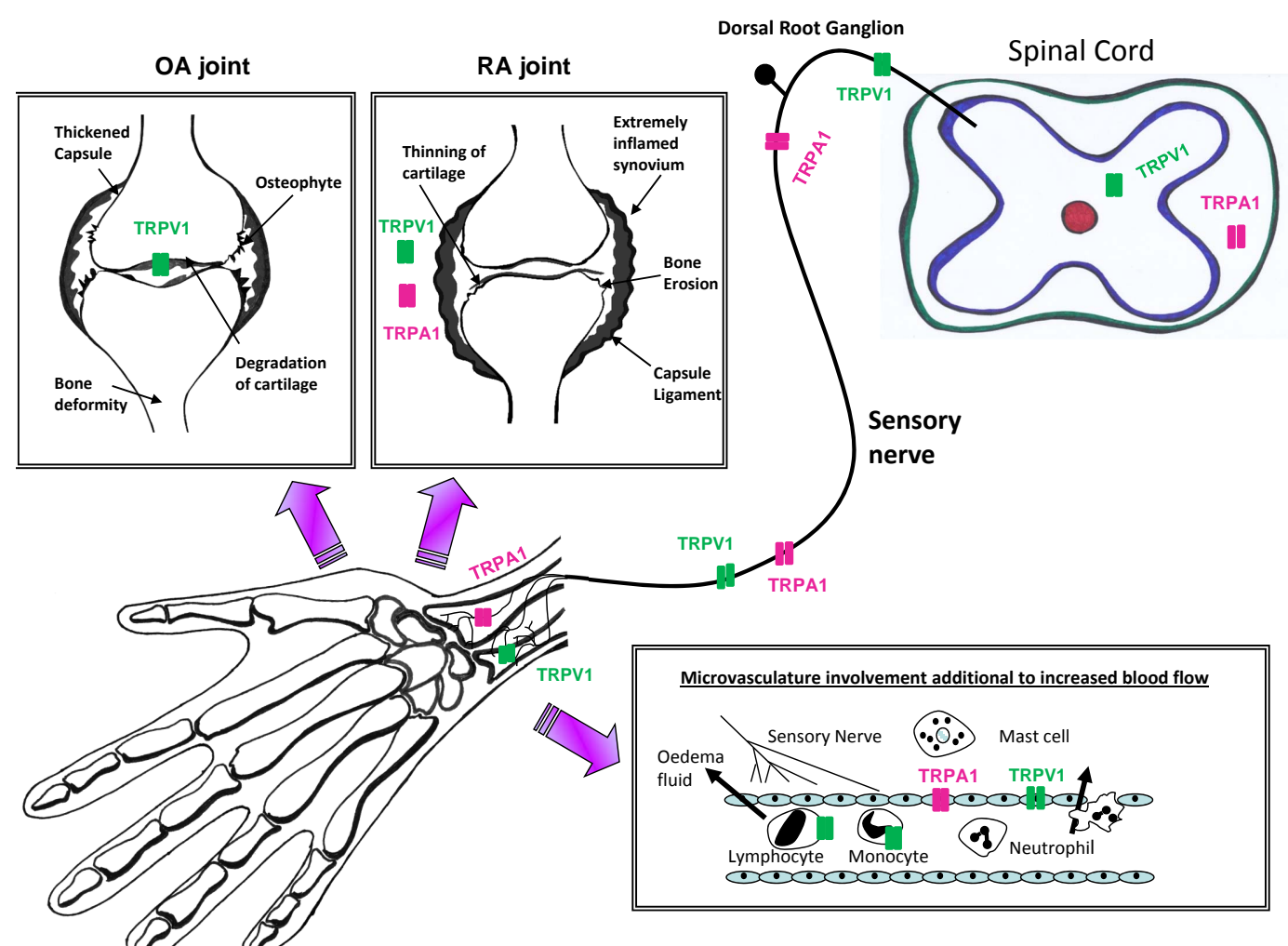

Fig. (1). TRPV1 and TRPA1 expression in arthritis. TRPV1 and TRPA1 play an important role on pain sensation in arthritis, with TRPV1 also contributing to the inflammation observed in RA. At present, TRPV1 (green) is known to be localized in OA chondrocytes, synoviocytes, DRGs, spinal cord, peripheral sensory nerves, endothelial cells and mononuclear cells. Evidence to date suggests that TRPA1 (pink) is expressed in synoviocytes, DRGs, spinal cord, peripheral sensory neurons and endothelial cells. TRPC5 is found in RA synoviocytes whilst TRPV4 is found in OA osteoblasts. Other TRP channels such as TRPC6, TRPC1 and TRPC3, TRPM2, TRPM3, TRPM7 and TRPV2 have also been found in the these cells although their roles in RA and OA remain to be elucidated.

\subsection{Clinical Relevance of Capsaicin}

The use of topical creams, lotions and patches to treat inflammatory and arthritic conditions has become widespread since the early 1980s. Currently, there are a range of creams available that contain various percentages of capsaicin. It has been difficult to directly associate the use of such creams with clinical effectiveness, thus clinical studies in this area are important. This is especially so when application of capsaicin-containing creams is associated with initial burning as a result of the stimulation of neurogenic inflammation and pain as discussed above [59, 60]. The benefits of capsaicin-induced neuronal desensitization for chronic neuropathic pain have been recently reviewed [53, 61]. Thus, this section of the review will focus on discussing the use of capsaicin-based creams for the treatment of arthritis.

McCarthy and McCarty [59] were one of the primary investigators of the effects of capsaicin in human arthritis. In their work, patients received topical capsaicin at $0.075 \%$ for 4 weeks (4 times a day), which was shown to alleviate the pain (tenderness) in the hand of patients with OA but not RA. These findings showed that although topical capsaicin caused initial pain; its chronic use could be beneficial for the treatment of OA-related pain in humans. As the initial pain caused by capsaicin creams was demonstrated to be a negative aspect of its usage by patients, a study by Schnitzer and collaborators [62] investigated the possibility of developing a maintenance regime for capsaicin treatment with reduced frequency of applications. Twice a day topical application of capsaicin cream $(0.25 \%)$ was as effective as a 4 times a day in alleviating the pain in the hands of OA patients. Importantly, capsaicin-treated patients showed reduced joint swelling, greater movement and grip strength elevated by $30 \%$ in week three just under double compared to placebotreated patients. Thus, a less frequent treatment with capsaicin cream resulted in an effective reduction of OArelated pain and inflammation whilst causing less discomfort for the patient [62]. Furthermore, topical application of capsaicin cream $(0.075 \%)$ on the knee of RA patients for 4 weeks reduced the synovial levels of SP, in addition to prostaglandin $\mathrm{E}_{2}\left(\mathrm{PGE}_{2}\right)$ and interleukin-6 (IL-6) which are well established mediators of RA [63].

These studies show that capsaicin creams, at different concentrations, are effective at relieving arthritic pain. More recently, a $8 \%$ capsaicin patch has been developed for the treatment of neuropathic pain and it has been licensed for use in Europe and United States of America (Qutenza; [64]). Indeed, clinical trials demonstrated that a single 60-minute of the $8 \%$ patch attenuated neuropathic pain for up to 12 weeks [53]. It is thought that this high concentration of capsaicin seems to cause a quicker neuronal desensitization and to have longer term analgesic effects [53]. It remains to 
Table 2. Endogenous Activators of TRPV1 and Their Role in Arthritis

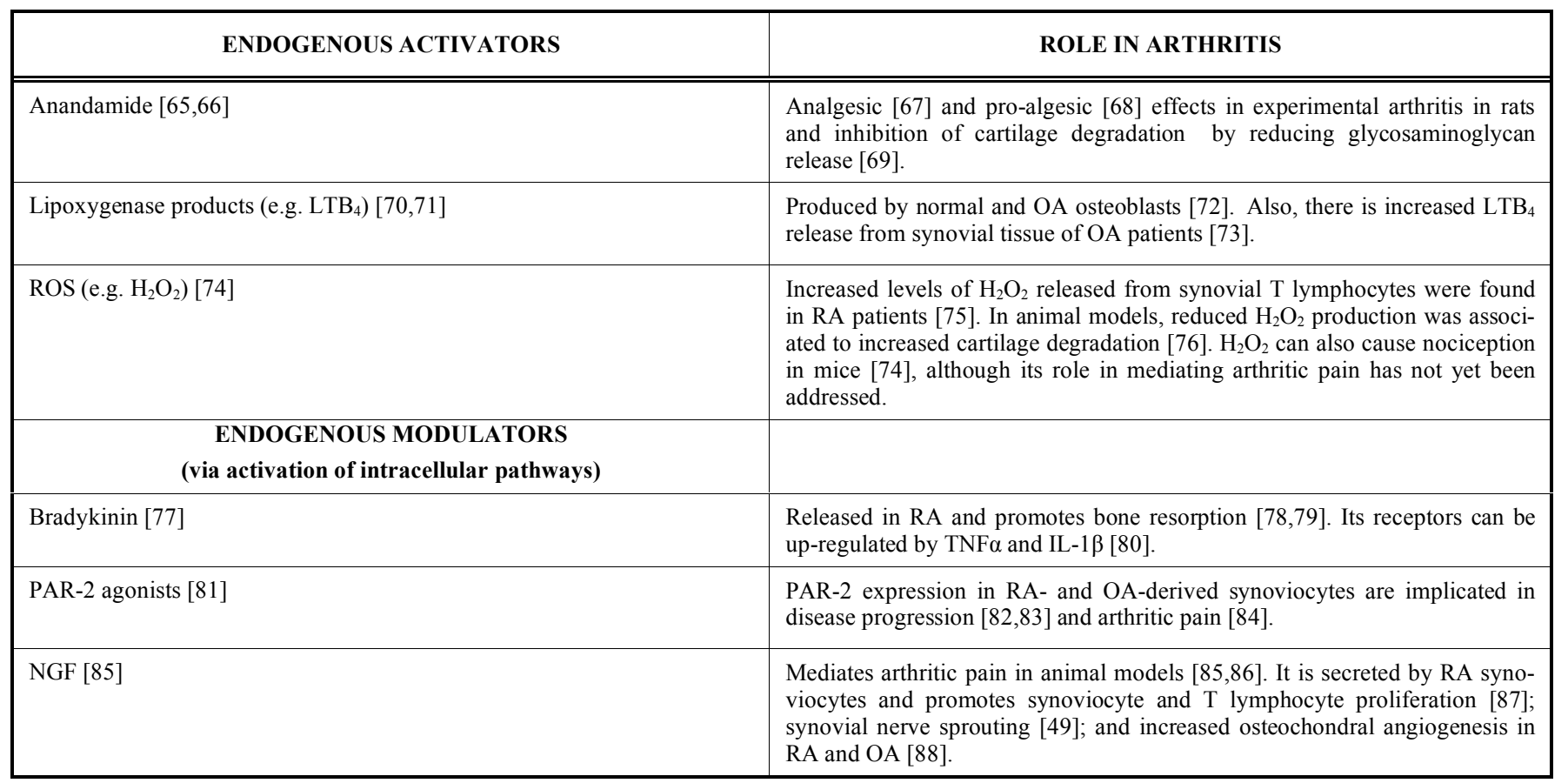

be investigated if the same formulation will be an effective therapy for arthritic pain.

\subsection{TRPV1}

Capsaicin selectively stimulates the primary afferents by activating and binding to TRPV1 that was cloned in 1997 [7]. TRPV1 is a ligand-gated, non-selective ion channel that functions as an integrator of multiple nociceptive stimuli including heat $\left(>43^{\circ} \mathrm{C}\right)$, low $\mathrm{pH}$, and a variety of putative endogenous lipid ligands, in addition to capsaicin. Indeed, endogenous TRPV1 agonists and modulators are known to play an active role in arthritis and are summarized in Table 2. Thus, the fact that capsaicin treatment alleviates pain and inflammation in arthritis; in addition to TRPV1 being activated by a variety of inflammatory stimuli and the wide expression of this channel in different tissues and cells, makes it an attractive drug development target for the treatment of arthritic conditions. Here, we discuss the importance of TRPV1 expression in the context of arthritis.

TRPV1 positive sensory nerves are present in the mouse knee and ankle joints [89]. Combined studies of tracing and immunohistochemistry revealed that approximately $40 \%$ of articular afferent nerves are TRPV1 positive and most of them also contain classic neuropeptides. The release of neuropeptides within the joint would be expected to cause dilatation. Perhaps surprisingly though, in the mouse knee joint, the TRPV1 agonist capsaicin mediates vasoconstriction [90]. This response has also been observed in certain vascular tissues and is not abolished by denervation [91]. This suggests that the ability of capsaicin to mediate vasoconstriction may be due to the presence of non-neuronal TRPV1 receptors. Besides being expressed on sensory neurons, there is evidence for functional TRPV1 receptor channels on synoviocytes [92, 93] and glial cells [94] in addition to blood vessels. Recently, TRPV1 was found to mediate cell death in rat synovial fibroblasts through calcium entry-dependent ROS production and mitochondrial depolarization [95]. Moreover, it has been proposed that the microRNA (miR-146a) plays an important role in OA by modulating inflammatory mediators such as TNF $\alpha$, IL-6 and IL- 8 as well as pain-related molecules such as TRPV1 in human glial cells, perhaps playing a pivotal role in influencing knee joint homeostasis and arthritic pain through effects on glial cells [96]. Thus, the potential exists for both neuronal and non-neuronal localisation of TRPV1 contributing to pain and inflammation [for review see: 97, 98].

Similarly to capsaicin, TRPV1 activation by other agonists triggers the release of CGRP and SP. Whilst it is now realised that substance $P$ is unlikely to influence the arthritic disease process in humans, the role of CGRP is less well understood. CGRP acts through vasodilation of vessels, which causes redness and warmth at the surface $[99,100]$ in different tissues including in the joint [101]. CGRP, like substance $\mathrm{P}$, may also influence endothelial cell proliferation in the joint [102] although the relevance of this is currently unknown. It has been suggested that blocking CGRP may alleviate pain in temporomandibular joint disorder [103]. This is of interest as it is known that the trigeminal ganglia is involved in CGRP-mediated pain processing in migraine, where CGRP antagonists have been shown to be of benefit in clinical trials [104].

\subsection{TRPV1 Knockout Mice (TRPV1KO) and Investigations in Inflammatory Joint Inflammation/Pain}

TRPV1KO mice were established shortly after the cloning of the TRPV1 receptor. The TRPV1 gene was disrupted by deleting an exon encoding part of the fifth and all of the sixth putative transmembrane domains of the channel, together with the intervening pore-loop region, meaning the TRPV1 receptor is expressed on the cell 
membrane, but it is not active [8]. They grow and reproduce normally. Importantly, a selective loss of thermal hypersensitivity during inflammatory responses was shown $[8,105]$ and diminished joint inflammation and pain in models of CFA-induced arthritis in the TRPV1 KO mouse, when compared with WT mouse [9]. This study was complemented by that of Keeble and collaborators [10] who showed that CFA-induced mono-arthritis was associated with less thermal hyperalgesia and knee swelling, despite TNF $\alpha$ levels remaining similarly high in WT and TRPV1 $\mathrm{KO}$ mice. In addition, another group showed by using a similar model of inflammatory pain that TRPV1KO mice experience less joint damage and altered weight distribution [11].

Mechanistic studies indicated that $\mathrm{TNF} \alpha$, present in high levels in the WT and TRPV1 KO mouse, induces thermal hyperalgesia via a TRPV1-dependent mechanism that also involved cyclooxygenase products and other cytokines such as IL-1 $\beta$ [15]. It was suggested that there are a series of peripheral events through which TNF $\alpha$ triggers and mediates inflammatory pain in diseases with a symmetrical aspect such as arthritis. A study carried out by Chen and collaborators in 2009 [14], aimed to elucidate if TRPV1 receptors were able to activate spinal cord glial cells, and therefore allowing the assumption that this channel was involved in pain stimulation. They showed TRPV1 KO mice did not develop hypersensitivity in adjuvant-induced arthritis as expected when compared to WT littermates. However, immunohistochemical analysis revealed that TRPV1 receptors are able to activate spinal glia, in the WT but not TRPV1 KO mice, suggesting a direct relationship with the observed pathological pain and inflammation [14]. Moreover, it was later demonstrated that this was associated with ERK signalling [13]. Similar findings were described by Honore and collaborators also using TRPV1 KO mice [21]. In their study, motor function was measured using photo-beam activity monitors and movement apparatus in addition to thermal sensitivity and calcium concentrations in vitro. Recently, it was established that central, but not peripherally expressed TRPV1, plays a role in the bilateral mechanical hyperalgesia induced by TNF $\alpha$ [17].

The studies, involving TRPV1 KO mice, are under a situation where the receptor deletion was life-long. This is of course different to the normal situation in animal and humans that develop arthritis. However, they provide evidence towards the hypothesis that TRPV1 antagonists may be beneficial in the treatment of arthritis. Moreover, this concept is supported by the knowledge that capsaicin creams that act, initially at least, via the TRPV1 receptor are beneficial in the human.

\subsection{TRPV1 Antagonists and Inflammatory Inflammation/Pain, Relevant to Joint Inflammation and RA}

The studies above demonstrate the involvement of the TRPV1 receptor in pain and inflammation associated with arthritis. Without doubt, these results heighten interest in the probability that TRPV1 antagonists would also be beneficial. Indeed, there have been key demonstrations that suggest TRPV1 antagonists have beneficial effects in relieving the symptoms associated with arthritis [106].
The TRPV1 antagonist, A-425619 was shown to reduce complete Freund's adjuvant-induced pain. It also alleviated pain after either i.t. administration or local injection into the inflamed paw [18], demonstrating the potential of TRPV1 antagonists. Furthermore Lappin and co-workers [19] showed that the TRPV1 antagonist SB366791 additionally inhibited glutamatergic neuro-transmission following CFAinduced peripheral inflammation. A study carried out by Kanai et al., [20] explored two TRPV1 antagonists (SB366791 and BCTC) in a CFA model of adjuvant-induced arthritis in the rat. They suggested that the antagonists inhibited thermal, but not mechanical hyperalgesia when given locally. However, the antagonists inhibited the mechanical hyperalgesia when given intrathecally, thus supporting the concept that central, in addition to peripheral TRPV1 mechanisms are important for pain sensation [17, 20].

Honore and collaborators [21] showed that the potent and selective TRPV1 antagonist, ABT-102 (Abbott), is an effective agent in blocking thermal and mechanical pain in a range of models. Importantly, there was an increase in analgesic efficacy with repeated administration of ABT-102 for 5-12 days. These beneficial effects were seen also with a structurally distinct TRPV1 antagonist, A-993610 [21]. By this time it was realised that TRPV1 antagonists induced an adverse hyperthermia. However, ABT-102 had a mild effect on core body temperature that appeared tolerated [21]. The authors concluded that the analgesic efficacy of ABT-102 supported its testing in clinical studies.

\subsection{Research using Models for OA and other Arthritic Conditions}

There is evidence for TRPV1 up-regulation in models of $\mathrm{OA}$ [107] and increased joint innervations in rats treated intra-articularly with monoiodoacetate (MIA) involving CGRP positive sensory neurons [108]. The TRPV1 receptor antagonist, A-889425, has been investigated in this model of OA. Effects on grip behaviour and firing of neurons were studied. A-889425 (10-300 $\mu \mathrm{mol} / \mathrm{kg}$, p.o.) attenuated loss of grip force and this was associated with reduced mechanical sensitivity. In addition, the increase in spontaneous firing following OA induction was reduced by A-889425 treatment [22]. Repeated treatment with another TRPV1 antagonist, A995662, was also effective in attenuating the MIA-induced osteoarthritic pain in rats. This antagonist was suggested to inhibit glutamate and CGRP release from the spinal cord [109]. Similarly, the TRPV1 antagonist ABT-102 attenuated pain in a model of osteoarthritic pain induced by MIA [21]. These results provide pre-clinical evidence of a potential role for TRPV1 antagonists in treating OA.

There are, as discussed in the introduction, many types of arthritis and the intense pain associated with episodes of gout are well known. Gout is induced by deposition of monosodium urate crystals in joints and this can be modelled in rats. Recently, it was shown that treatment with the selective TRPV1 receptor antagonists SB366791 or AMG9810 substantially inhibited the pain sensitivity and the swelling (oedema formation) observed in response to monosodium urate injection. Whilst a range of other mediators are involved, this work cited the importance of mast cell-dependent mechanisms with the clear involvement 
of TRPV1 [110]. Thus there may be a use for TRPV1 antagonists alleviating the sharp pain associated with gout.

\subsection{Clinical Trials}

The efficacy of TRPV1 antagonists in pre-clinical models is supported by the fact that there have been at least 7 antagonists that have progressed to clinical trials, where one of the first, SB-705498, progressed from phase 1 trials a number of years ago [106]. However, to our knowledge, the efficacy of a TRPV1 antagonist in human arthritis has yet to be published. It is difficult to know at this stage, whether this is due to the delay in obtaining suitable drugs for humans, or whether clinical trials have been performed, but the data is at present unpublished.

\section{EVIDENCE FOR THE INVOLVEMENT OF OTHER TRP CHANNELS IN ARTHRITIS}

In recent years there has been exciting basic research into the pathophysiological relevance of other TRP channels that have been shown to be expressed in different cells and possess biological functions. The new research aims to understand the importance of these channels for pain regulation and inflammation, including arthritis.

\subsection{TRPA1}

The transient receptor potential ankyrin 1 (TRPA1), in addition to TRPV1, has been shown to play a role in mediating inflammatory pain. TRPA 1 was first cloned in 1999 [24] and it is known to be expressed in the same sensory neurons as TRPV1, and activated by a range of endogenous and exogenous agonists (see: [98]). This review will highlight the importance of TRPA1 activation by cold [111] and by endogenous agonists for arthritis. TRPA1 endogenous activators are often electrophilic compounds such as reactive oxygen species $[112,113]$ and lipid mediators such as 15d-PGJ2 [114]100; which are generated in arthritis [115-118].

Research using rodent models has revealed the role of TRPA1 role in different pain models which have been recently reviewed [119-121]. However, little is known of the specific roles of TRPA1 in arthritis. In addition to TRPA1 expression in sensory neurons, TRPA1 alongside TRPV1 is found expressed in non-neuronal cells such as rat endothelial cells [122] and human synoviocytes [92]. TRPA1 expression in synoviocytes and endothelial cells is functional, i.e.; elicits calcium influx when stimulated $[92,122]$; suggesting this receptor could be an important mediator of joint inflammation and pain. It is only recently, with the generation of TRPA1KO mice and selective TRPA1 antagonists, that the role of this channel in arthritic pain has been explored. These studies have used models of inflammatory pain which resemble RA and models of OA.

In 2007, Petrus and collaborators [27] published the first evidence of a selective TRPA1 antagonist being effective in reducing arthritic pain. By using a model of i.pl. CFAinduced inflammatory pain, co-injection with the selective TRPA1 antagonist AP-18 abolished mechanical hyperalgesia in mice. The same study showed TRPA1 KO mice exhibited normal mechanical hyperalgesia $24 \mathrm{~h}$ following CFA injection. Recently, our group developed a model of bilateral mechanical hyperalgesia induced by i.pl. TNF $\alpha$, a model of pain relevant for the study of arthritis [17]. We found that both intrathecal (i.t.) and intraplantar (i.pl.) administration of a selective TRPA1 antagonist, AP-18, caused analgesia. This analgesic effect was more pronounced in animals treated with i.pl. AP-18, suggesting an important role for these receptors at a peripheral level, in contrast with a central role for TRPV1 receptors observed in the same model. This research showed for the first time that although these receptors are expressed in the same sensory neurons, they play specific and additional roles in the mediation of pain. In addition, the same study showed that TRPA1 KO mice exhibited analgesia when treated either with i.pl. TNF $\alpha$ or intrarticular CFA, in comparison with WT littermates. We highlight here that CFA-induced pain was decreased in TRPA1 KO mice only from 1 week after CFA injection. These results together with those described by Petrus et al. [27] suggest a primary role for TRPA1 in the maintenance of mechanical hyperalgesia in inflammatory states. Similarly, a study performed in rats [28] showed the oral effects of another selective TRPA1 antagonist HC-030031 in reducing the mechanical hyperalgesia caused by CFA in the paw. In addition, systemic administration of the selective TRPA1 antagonist A-967079 in rats injected i.pl. with CFA, reduced the activity of spinal wide dynamic range neurons after noxious mechanical stimuli such as $10 \mathrm{~g}$ von Frey hairs and noxious pinch stimulation [29]. Interestingly, TRPA1 expression is increased in small and medium size DRG neurons following i.pl. CFA [123].

Although the participation of TRPA1 in mediating pain in models of RA is clear, its role in OA is still of debate. To date, there are only 2 reports of studies aimed at elucidating TRPA1 involvement in MIA-induced OA-related pain. The first performed in 2010 by McGaraughty and collaborators [29], showed a reduced neuronal activity in rats systemically treated with A-967079 when tested for high-intensity von Frey hair stimulation $(300 \mathrm{~g})$ and compared to vehiclecontrols. On the other hand, the study of Okun and collaborators [23] which investigated the participation of TRPA1 in ongoing pain, showed that neither systemic nor i.pl. treatment with the selective TRPA1 antagonist HC030031 was able to interfere with weight bearing responses caused by intra-articular MIA. These differences may be due to experimental models suggesting that TRPA1 may play a role in OA-related pain when a mechanical stimulus is involved but not continuous pain.

As mentioned before, TRPA1 can be activated by cold $\left(10-17^{\circ} \mathrm{C}\right)$. The relationship between cold exposure and increased pain in arthritic joints has been suggested [27, 124, 125] and thus, it is sensible to speculate if TRPA1 plays a role in this increased sensitivity. Few studies have addressed this field but it has now been described that although TRPA1's role in sensing cold in physiological conditions is conflicting (see: $[121,126]$ ), that a role for TRPA1 in coldinduced responses in animals with pre-established inflammation is evident. Indeed, Petrus and collaborators [27] showed that co-injection with AP-18 reduces CFAinduced cold hyperalgesia $\left(5^{\circ} \mathrm{C}\right)$ in the rat paw. In a different experimental setting, cold hyperalgesia was tested in mice with their CFA-treated paws (i.pl.) sprayed with tetrafluorethane that is known to cause licking behaviour. This response was reduced by systemic treatment with HC030031 [30]. A study performed by Obata and collaborators 
[123] showed that TRPA1 anti-sense knockdown causes a reduction in cold hyperalgesia induced by CFA i.pl. injection in rats, although the same treatment failed to prevent the thermal and mechanical hyperalgesia triggered by CFA. Overall, these results suggest in a broad sense, a link between cold sensation and inflammation in RA. On the other hand, the mechanisms underlying this relationship remain unclear.

Although there is clear evidence from animal models that TRPA1 plays a role in the mechanical and cold responses related to arthritis, the potential use of TRPA1 antagonists to treat human pain remains to be addressed. In addition, research into the role of TRPA1 channels in arthritis is still novel and very little is understood of TRPA1 participation in arthritis. Plus, the identification of the specific endogenous agonists playing a role at different stages of RA and OA would be extremely important. Thus, there is still a great need of fundamental mechanistic studies in order to fully understand the role of TRPA1 channels in both RA and OA. Studies from our laboratory have revealed the distinct roles of TRPV1 and TRPA1 in a single study. This highlights the potential that a combined treatment of TRPV1 and TRPA1 antagonists may be a more beneficial therapeutic approach than inhibiting with of these TRP receptors alone.

\subsection{Other TRP Channels}

A potential role for other TRP channels, in addition to TRPV1 and TRPA1, in arthritis has been speculated. Perhaps, the most compelling evidence is in regards to TRPV4 and TRPC5 (transient receptor potential canonical 5). In addition to being neuronally expressed [127, 128], both receptors are functionally expressed in human synoviocytes either derived from commercial cell lines or obtained from RA patients $[92,129,130]$.

Indeed, the suggested role of TRPC5 in pain and RA is very new. This channel was first described as an important regulator of brain development [131]. Very recently, this receptor channel was revealed as a peripheral cold sensor in naive mice [132]. Interestingly, treatment of RA-derived fibroblast-like synoviocytes (FLS) with either an antiTRPC5 antibody or the knockdown of TRPC1/5 expression, increases metalloproteinase expression (MMP-2, -9 and -1) [129]; suggesting TRPC5 plays a possible protective role in RA. Thioredoxin is one of the endogenous activators of TRPC5 and has been suggested to play a role in arthritis. The reduced thioredoxin form is able to break disulphide bridges between cysteine residues and activate TRPC5 [129]. Systemic thioredoxin treatment was shown to reduce arthritis severity in a murine model of RA induced by $\mathrm{mAb} / \mathrm{LPS}$ injection. The same study described a protective response to arthritis in thioredoxin overexpressing transgenic (TG) mice [133]. In addition, in vitro studies showed that thioredoxin inhibits MMP release from FLS, an effect that was reduced in the presence of an anti-TRPC5 antibody [129]. Thioredoxin has been also linked to innate immune cell regulation (neutrophils and macrophages) and inhibition of neovascularisation [134], as well acting as a co-stimulatory molecule for IL-6 and IL-8 production in FLS treated with $\mathrm{TNF} \alpha[135]$.
TRPV4, on the other hand, has been primarily implicated in OA. TRPV4 is a sensor for warmer temperatures (30$34^{\circ} \mathrm{C}$; [136]) and in addition to its expression in neurons and synoviocytes, TRPV4 protein has been localised to in mouse chondrocytes [137]. The same study showed that TRPV4 deletion in mice is associated to a severe, progressive OA at a young age, particularly in male mice, accompanied by significant increases in ossification of joint tissues. Also, TRPV4 deletion results in a reduction of osmotic sensitivity by chondrocytes, which in turn could result in decreased adaptative changes in response to cartilage overloading and degeneration. The collected evidence suggests a chondroprotective role for this channel in OA.

Other TRP channels such as TRPC6, TRPC1 and TRPC3, TRPM2 (transient receptor potential melastatin 2), TRPM3, TRPM7 and TRPV2 are also expressed in synoviocytes, chondrocytes, mononuclear cells and neutrophils [92, 138-140]; although their roles in RA and $\mathrm{OA}$ remain to be elucidated.

\section{CONCLUSION}

An American investigation into the mortality rates of arthritis, involving over 5000 individuals with RA, found that those with the condition had a mortality rate at least twice as high (if not more) than those without it [141-143]. These statistics allow us to further acknowledge the severity of arthritis. Moreover, it is established that arthritic patients are often unhappy with the level of pain relief that is achieved with currently available drugs [144]. The established drugs currently used can be divided into the main groups of those that relieve pain and those that alter the disease progression [145]. Those involved in pain relief with a potential for little anti-inflammatory relief are the analgesics or painkillers like dihyrocodeine and paracetamol. The non-steroidal anti-inflammatory drugs (NSAIDs), inhibitors of cyclo-oxygenase, (e.g. proxens) work to combat the pain and are also classed as anti-inflammatory. Within these groups we would also place the capsaicin creams and patches. The NSAIDs are extremely widely used, but do not halt disease progression in rheumatoid arthritis. Also there are problems with dependency (opioids) liver adverse effects (paracetamol) and internal bleeding (systemic cyclooxygenase inhibitors). Thus, potentially TRPV1 and TRPA 1 antagonists may be as effective, whilst also having less serious side-effects. Other drugs such as the corticosteroids (e.g. prednisone, the most commonly prescribed corticosteroid) work to control the inflammation and can be injected locally, but they have to be used with care. Finally, there is a wealth of disease modifying anti-rheumatic drugs available, of which methotrexate is the best known and here we also include the biologics (antibody therapy), that are used alone or in combination to attenuate or halt the progression of arthritis. Like many drugs, these medicines currently used for arthritis can have undesirable side effects. However, it is highly unlikely, from present knowledge, that TRP-related drugs can influence the immune aspect of these drugs in a similar manner. On the other hand, without doubt there is an unmet need for effective pain killers and it is here that the TRP-related drugs fit best when using the current knowledge, discussed in this review. They may be suitable for several applications including topical, in addition to 
systemic with a potential for use in combination therapies, in addition to their use alone.

Without doubt, the TRPV1 cation channels have been clearly demonstrated to play a key role in pain perception. This understanding has given new optimism in finding novel possible treatments for chronic pain diseases like arthritis, especially OA that has currently fewer treatment options. This has been particularly so with respect to TRPV1 antagonists and we believe this optimism should be extended to other receptors, specifically TRPA1.

\section{CONFLICT OF INTEREST}

The authors confirm that this article content has no conflicts of interest.

\section{ACKNOWLEDGEMENTS}

We thank Arthritis Research UK and these studies were supported by a Capacity Building Award in Integrative Mammalian Biology funded by the BBSRC, BPS, HEFCE, KTN, MRC and SFC.

\section{REFERENCES}

[1] Rantapää-Dahlqvist S, de Jong BA, Berglin E, et al. Antibodies against cyclic citrullinated peptide and $\operatorname{IgA}$ rheumatoid factor predict the development of rheumatoid arthritis. Arthritis Rheum 2003; 48: 2741-9.

[2] Anderson J, Caplan L, Yazdany J, et al. Rheumatoid arthritis disease activity measures: american college of rheumatology recommendations for use in clinical practice. Arthritis Care Res (Hoboken) 2012; 64: 640-7.

[3] Gardner DL. The nature and causes of osteoarthrosis. Br Med J 1983; 286: 418-24.

[4] Wang M, Shen J, Jin H, et al. Recent progress in understanding molecular mechanisms of cartilage degeneration during osteoarthritis. Ann N Y Acad Sci 2011; 1240: 61-9.

[5] Colmegna I, Ohata BR, Menard HA. Current understanding of rheumatoid arthritis therapy. Clin Pharmacol Ther 2012; 91: 60720.

[6] Hunter DJ. Osteoarthritis. Best Pract Res Clin Rheumatol 2011; 25: 801-14.

[7] Caterina MJ, Schumacher MA, Tominaga M, et al. The capsaicin receptor: a heat-activated ion channel in the pain pathway. Nature 1997; 389: 816-24.

[8] Caterina MJ, Leffler A, Malmberg AB, et al. Impaired nociception and pain sensation in mice lacking the capsaicin receptor. Science 2000; 288: 306-13.

[9] Szabó A, Helyes Z, Sándor K, et al. Role of transient receptor potential vanilloid 1 receptors in adjuvant-induced chronic arthritis: in vivo study using gene-deficient mice. J Pharmacol Exp Ther 2005; 314: 111-9.

[10] Keeble J, Russell F, Curtis B, et al. Involvement of transient receptor potential vanilloid 1 in the vascular and hyperalgesic components of joint inflammation. Arthritis Rheum 2005; 52: 3248-56.

[11] Barton NJ, McQueen DS, Thomson D, et al. Attenuation of experimental arthritis in TRPV1R knockout mice. Exp Mol Pathol 2006; 81: 166-70.

[12] Chen Y, Willcockson HH, Valtschanoff JG. Increased expression of CGRP in sensory afferents of arthritic mice--effect of genetic deletion of the vanilloid receptor TRPV1. Neuropeptides 2008; 42: 551-6.

[13] Chen Y, Willcockson HH, Valtschanoff JG. Vanilloid receptor TRPV1-mediated phosphorylation of ERK in murine adjuvant arthritis. Osteoarthr Cartil 2009; 17: 244-51.

[14] Chen Y, Willcockson HH, Valtschanoff JG. Influence of the vanilloid receptor TRPV1 on the activation of spinal cord glia in mouse models of pain. Exp Neurol 2009; 220: 383-90.

[15] Russell FA, Fernandes ES, Courade JP, et al. Tumour necrosis factor alpha mediates transient receptor potential vanilloid 1dependent bilateral thermal hyperalgesia with distinct peripheral roles of interleukin-1beta, protein kinase $\mathrm{C}$ and cyclooxygenase-2 signalling. Pain 2009; 142: 264-74.

[16] Willcockson HH, Chen Y, Han JE, et al. Effect of genetic deletion of the vanilloid receptor TRPV1 on the expression of substance $\mathrm{P}$ in sensory neurons of mice with adjuvant-induced arthritis. Neuropeptides 2010; 44: 293-7.

[17] Fernandes ES, Russell FA, Spina D, et al. A distinct role for transient receptor potential ankyrin 1, in addition to transient receptor potential vanilloid 1 , in tumor necrosis factor $\alpha$-induced inflammatory hyperalgesia and Freund's complete adjuvantinduced monarthritis. Arthritis Rheum 2011; 63: 819-29.

[18] Honore P, Wismer CT, Mikusa J, et al. A-425619 [1-isoquinolin-5yl-3-(4-trifluoromethyl-benzyl)-urea], a novel transient receptor potential type $\mathrm{V} 1$ receptor antagonist, relieves pathophysiological pain associated with inflammation and tissue injury in rats. J Pharmacol Exp Ther 2005; 314: 410-21.

[19] Lappin SC, Randall AD, Gunthorpe MJ, et al. TRPV1 antagonist, SB-366791, inhibits glutamatergic synaptic transmission in rat spinal dorsal horn following peripheral inflammation. Eur J Pharmacol 2006; 540: 73-81.

[20] Kanai Y, Hara T, Imai A, et al. Differential involvement of TRPV1 receptors at the central and peripheral nerves in CFA-induced mechanical and thermal hyperalgesia. J Pharm Pharmacol 2007; 59: 733-8.

[21] Honore P, Chandran P, Hernandez G, et al. Repeated dosing of ABT-102, a potent and selective TRPV1 antagonist, enhances TRPV1-mediated analgesic activity in rodents, but attenuates antagonist-induced hyperthermia. Pain 2009; 142: 27-35.

[22] Chu KL, Chandran P, Joshi SK, et al. TRPV1-related modulation of spinal neuronal activity and behavior in a rat model of osteoarthritic pain. Brain Res 2011; 1369: 158-66.

[23] Okun A, Liu P, Davis P, et al. Afferent drive elicits ongoing pain in a model of advanced osteoarthritis. Pain 2012; 153: 924-33.

[24] Jaquemar D, Schenker T, Trueb B. An ankyrin-like protein with transmembrane domains is specifically lost after oncogenic transformation of human fibroblasts. J Biol Chem 1999; 274: 7325 33 .

[25] Bautista DM, Jordt SE, Nikai T, et al. TRPA1 mediates the inflammatory actions of environmental irritants and proalgesic agents. Cell 2006; 124: 1269-82.

[26] Kwan KY, Allchorne AJ, Vollrath MA, et al. TRPA1 contributes to cold, mechanical, and chemical nociception but is not essential for hair-cell transduction. Neuron 2006; 50: 277-89.

[27] Petrus M, Peier AM, Bandell M, et al. A role of TRPA1 in mechanical hyperalgesia is revealed by pharmacological inhibition. Mol Pain 2007; 3: 40.

[28] Eid SR, Crown ED, Moore EL, et al. HC-030031, a TRPA1 selective antagonist, attenuates inflammatory- and neuropathyinduced mechanical hypersensitivity. Mol Pain 2008; 4: 48.

[29] McGaraughty S, Chu KL, Perner RJ, et al. TRPA1 modulation of spontaneous and mechanically evoked firing of spinal neurons in uninjured, osteoarthritic, and inflamed rats. Mol Pain 2010; 6: 14.

[30] da Costa DS, Meotti FC, Andrade EL, et al. The involvement of the transient receptor potential A1 (TRPA1) in the maintenance of mechanical and cold hyperalgesia in persistent inflammation. Pain 2010; 148: 431-7.

[31] Thresh LT. Isolation of capsaicin. Pharm J 1846; 6: 941.

[32] Nelson EK. The constitution of capsaicin the pungent principle of capsicum. J Am Chem Soc 1919; 41: 1115-7.

[33] Szolcsányi J, Jancsó-Gábor. Sensory effects of capsaicin congeners I. Relationship between chemical structure and pain producing potency of pungent agents. Arzneimittelforschung. 1975b; 25: 1877-81.

[34] Szolcsányi J. A pharmacological approach to elucidation of the role of different nerve fibres and receptor endings in mediation of pain. J Physiol (Paris) 1977; 73: 251-9.

[35] Caterina MJ, Rosen TA, Tominaga M, et al. A capsaicin-receptor homologue with a high threshold for noxious heat. Nature 1999; 398: 436-41.

[36] Brain SD, Cox HM. Neuropeptides and their receptors: innovative science providing novel therapeutic targets. Br J Pharmacol 2006; 147: S202-11.

[37] Colpaert FC, Donnerer J, Lembeck F. Effects of capsaicin on inflammation and on the substance $P$ content of nervous tissues in rats with adjuvant arthritis. Life Sci 1983; 32: 1827-34. 
[38] Levine JD, Moskowitz MA, Basbaum AI. The contribution of neurogenic inflammation in experimental arthritis. J Immunol 1985; 135: 843s-7 s.

[39] Ferrell WR, Russell NJ. Extravasation in the knee induced by antidromic stimulation of articular $\mathrm{C}$ fibre afferents of the anaesthetized cat. J Physiol 1986; 379: 407-16.

[40] Liu D, Jiang LS, Dai LY. Substance P and its receptors in bone metabolism. Neuropeptides 2007; 41: 271-83.

[41] McDougall JJ, Hanesch U, Pawlak M, et al. Participation of NK1 receptors in nociceptin-induced modulation of rat knee joint mechanosensitivity. Exp Brain Res 2001; 137: 249-53.

[42] Pawlak M, Schmidt RF, Heppelmann B, et al. The neurokinin-1 receptor antagonist RP 67580 reduces the sensitization of primary afferents by substance $\mathrm{P}$ in the rat. Eur J Pain 2001; 5: 69-79.

[43] Goldstein DJ, Wang O, Saper JR, et al. Ineffectiveness of neurokinin-1 antagonist in acute migraine: a crossover study. Cephalalgia 1997; 17: 785-90

[44] Hill R. NK1 (substance P) receptor antagonists why are they not analgesic in humans? Trends Pharmacol Sci 2000; 21: 244-6.

[45] Urban LA, Fox AJ. NK1 receptor antagonists--are they really without effect in the pain clinic? Trends Pharmacol Sci 2000; 21: 462-4.

[46] Weihe E, Nohr D, Schäfer MK, et al. Calcitonin gene related peptide gene expression in collagen-induced arthritis. Can J Physiol Pharmacol 1995; 73: 1015-9.

[47] Bulling DG, Kelly D, Bond S, et al. Adjuvant-induced joint inflammation causes very rapid transcription of betapreprotachykinin and alpha-CGRP genes in innervating sensory ganglia. J Neurochem 2001; 77: 372-82.

[48] Bring DK, Heidgren ML, Kreicbergs A, et al. Increase in sensory neuropeptides surrounding the achilles tendon in rats with adjuvant arthritis. J Orthop Res 2005; 23: 294-301.

[49] Ghilardi JR, Freeman KT, Jimenez-Andrade JM, et al. Neuroplasticity of sensory and sympathetic nerve fibers in the painful arthritic joint. Arthritis Rheum 2012; 64970: 2223-32.

[50] Origuchi T, Iwamoto N, Kawashiri SY, et al. Reduction in serum levels of substance $\mathrm{P}$ in patients with rheumatoid arthritis by etanercept, a tumor necrosis factor inhibitor. Mod Rheumatol 2011; 21: 244-50.

[51] Porszasz J, Jancso N. Studies on the action potentials of sensory nerves in animals desensitized with capsaicin. Acta Physiol Acad Sci Hung 1959; 16: 299-306.

[52] Jancsó N, Jancsó-G, Szolcsányi J. Direct evidence for neurogenic inflammation and its prevention by denervation and by pretreatment with capsaicin. Br J Pharmacol Chemother 1967; 31: 138-51.

[53] Jancsó G, Király E, Jancsó-Gábor A. Pharmacologically induced selective degeneration of chemosensitive primary sensory neurones. Nature 1977; 270: 741-3.

[54] Knotkova H, Pappagallo M, Szallasi A. Capsaicin (TRPV1 Agonist) therapy for pain relief: farewell or revival? Clin J Pain 2008; 24: 142-54.

[55] Bley KR. TRPV1 agonist approaches for pain management. In: Gomtsyan A, Faltynek CR, Eds. Vanilloid receptor TRPV1 in drug discovery. targeting pain and other pathological disorders. Hoboken NJ, USA: John Willey and Sons Inc. 2010; pp. 325-47.

[56] Anand P, Bley K. Topical capsaicin for pain management: therapeutic potential and mechanisms of action of the new highconcentration capsaicin 8\% patch. Br J Anaesth 2011; 107: 490502 .

[57] Hara A, Sakurada T, Sakurada S, et al. Antinociceptive effects of neonatal capsaicin in rats with adjuvant arthritis. Naunyn Schmiedebergs Arch Pharmacol 1984; 326: 248-53.

[58] Helyes Z, Szabó A, Németh J, et al. Antiinflammatory and analgesic effects of somatostatin released from capsaicin-sensitive sensory nerve terminals in a Freund's adjuvant-induced chronic arthritis model in the rat. Arthritis Rheum 2004; 50:1677-8.

[59] McCarthy GM, McCarty DJ. Effect of topical capsaicin in the therapy of painful osteoarthritis of the hands. J Rheumatol 1992; 19: 604-7.

[60] Sawynok J. Topical analgesics in neuropathic pain. Curr Pharm Des 2005; 11:2995-3004

[61] Derry S, Lloyd R, Moore RA, et al. Topical capsaicin for chronic neuropathic pain in adults. Cochrane Database Syst Rev 2009; 4: CD007393.
[62] Schnitzer T, Morton C, Coker S. Topical capsaicin therapy for osteoarthritis pain: achieving a maintenance regimen. Semin Arthritis Rheum 1995; 23: 34-40.

[63] Weisman MH, Hagaman C, Yaksh TL, et al. Preliminary findings on the role of neuropeptide suppression by topical agents in the management of rheumatoid arthritis. Semin Arthritis Rheum 1994; 23: 18-24.

[64] McCormack PL. Capsaicin dermal patch: in non-diabetic peripheral neuropathic pain. Drugs 2010; 70: 1831-42.

[65] Zygmunt PM, Petersson J, Andersson DA, et al. Vanilloid receptors on sensory nerves mediate the vasodilator action of anandamide. Nature 1999; 400: 452-7.

[66] Smart D, Gunthorpe MJ, Jerman JC, et al. The endogenous lipid anandamide is a full agonist at the human vanilloid receptor (hVR1). Br J Pharmacol 2000; 129: 227-30.

[67] Smith FL, Fujimori K, Lowe J, et al. Characterization of delta9tetrahydrocannabinol and anandamide antinociception in nonarthritic and arthritic rats. Pharmacol Biochem Behav 1998; 60: 183-91.

[68] Gauldie SD, McQueen DS, Pertwee R, et al. Anandamide activates peripheral nociceptors in normal and arthritic rat knee joints. Br J Pharmacol 2001; 132: 617-21.

[69] Mbvundula EC, Bunning RA, Rainsford KD. Effects of cannabinoids on nitric oxide production by chondrocytes and proteoglycan degradation in cartilage. Biochem Pharmacol 2005; 69: 635-40.

[70] Hwang SW, Cho H, Kwak J, et al. Direct activation of capsaicin receptors by products of lipoxygenases: endogenous capsaicin-like substances. Proc Natl Acad Sci U S A 2000; 97 : 6155-60.

[71] Huang KS, Lin M, Cheng GF. Anti-inflammatory tetramers of resveratrol from the roots of Vitis amurensis and the conformations of the seven-membered ring in some oligostilbenes. Phytochemistry 2001; 58: 357-62.

[72] Paredes Y, Massicotte F, Pelletier JP, et al. Study of the role of leukotriene $\mathrm{B}_{4}$ in abnormal function of human subchondral osteoarthritis osteoblasts: effects of cyclooxygenase and/or 5lipoxygenase inhibition. Arthritis Rheum 2002; 46: 1804-12.

[73] Wittenberg RH, Willburger RE, Kleemeyer KS, et al. Prostaglandin and leukotriene release of synovial tissue in various joint diseases. Z Orthop Ihre Grenzgeb 1991; 129: 531-6.

[74] Keeble JE, Bodkin JV, Liang L, et al. Hydrogen peroxide is a novel mediator of inflammatory hyperalgesia, acting via transient receptor potential vanilloid 1-dependent and independent mechanisms. Pain 2009; 141: 135-42.

[75] Remans PH, van Oosterhout M, Smeets TJ, et al. Intracellular free radical production in synovial $\mathrm{T}$ lymphocytes from patients with rheumatoid arthritis. Arthritis Rheum 2005; 52: 2003-9.

[76] Schiller J, Benard S, Reichl S, et al. Cartilage degradation by stimulated human neutrophils: reactive oxygen species decrease markedly the activity of proteolytic enzymes. Chem Biol 2000; 7 : 557-68.

[77] Chuang $\mathrm{HH}$, Prescott ED, Kong $\mathrm{H}$, et al. Bradykinin and nerve growth factor release the capsaicin receptor from PtdIns(4,5)P2mediated inhibition. Nature 2001; 411: 957-62.

[78] Lerner UH, Jones IL, Gustafson GT. Bradykinin, a new potential mediator of inflammation-induced bone resorption. Studies of the effects on mouse calvarial bones and articular cartilage in vitro. Arthritis Rheum 1987; 30: 530-40.

[79] Ljunggren O, Lerner UH. Stimulation of bone resorption in cultured mouse calvaria by met-lys-bradykinin. J Periodontal Res 1988; 23: 75-7.

[80] Brechter AB, Persson E, Lundgren I, et al. Kinin $\mathrm{B}_{1}$ and $\mathrm{B}_{2}$ receptor expression in osteoblasts and fibroblasts is enhanced by interleukin-1 and tumour necrosis factor-alpha. Effects dependent on activation of NF-kappaB and MAP kinases. Bone 2008; 43: 7283.

[81] Amadesi S, Nie J, Vergnolle N, et al. Protease-activated receptor 2 sensitizes the capsaicin receptor transient receptor potential vanilloid receptor 1 to induce hyperalgesia. J Neurosci 2004; 24 : 4300-12.

[82] Amiable N, Martel-Pelletier J, Lussier B, et al. Proteinase-activated receptor-2 gene disruption limits the effect of osteoarthritis on cartilage in mice: a novel target in joint degradation. J Rheumatol 2011; 38: 911-20.

[83] Xue M, Chan YK, Shen K, et al. Protease-activated receptor 2 , rather than protease-activated receptor 1 , contributes to the 
aggressive properties of synovial fibroblasts in rheumatoid arthritis. Arthritis Rheum 2012; 64: 88-98.

[84] Helyes Z, Sándor K, Borbély E, et al. Involvement of transient receptor potential vanilloid 1 receptors in protease-activated receptor-2-induced joint inflammation and nociception. Eur J Pain 2010; 14: 351-8.

[85] Pezet S, Onténiente B, Jullien J, et al. Differential regulation of NGF receptors in primary sensory neurons by adjuvant-induced arthritis in the rat. Pain 2001; 90: 113-25.

[86] Shelton DL, Zeller J, Ho WH, et al. Nerve growth factor mediates hyperalgesia and cachexia in auto-immune arthritis. Pain 2005; 116: 8-16.

[87] Raychaudhuri SP, Raychaudhuri SK, Atkuri KR, et al. Nerve growth factor: a key local regulator in the pathogenesis of inflammatory arthritis. Arthritis Rheum 2011; 63: 3243-52.

[88] Walsh DA, McWilliams DF, Turley MJ, et al. Angiogenesis and nerve growth factor at the osteochondral junction in rheumatoid arthritis and osteoarthritis. Rheumatology (Oxford) 2010; 49: 185261.

[89] Cho WG, Valtschanoff JG. Vanilloid receptor TRPV1-positive sensory afferents in the mouse ankle and knee joints. Brain Res 2008; 1219: 59-65.

[90] Keeble JE, Brain SD. Capsaicin-induced vasoconstriction in the mouse knee joint: a study using TRPV1 knockout mice. Neurosci Lett 2006; 401: 55-8.

[91] Kark T, Bagi Z, Lizanecz E, et al. Tissue-specific regulation of microvascular diameter: opposite functional roles of neuronal and smooth muscle located vanilloid receptor-1. Mol Pharmacol 2008; 73: $1405-12$.

[92] Kochukov MY, McNearney TA, Fu Y, et al. Thermosensitive TRP ion channels mediate cytosolic calcium response in human synoviocytes. Am J Physiol Cell Physiol 2006; 291: C424-32.

[93] Kochukov MY, McNearney TA, Yin H, et al. Tumor necrosis factor-alpha (TNF-alpha) enhances functional thermal and chemical responses of TRP cation channels in human synoviocytes. Mol Pain 2009; $5: 49$.

[94] Chen Y, Willcockson HH, Valtschanoff JG. Influence of the vanilloid receptor TRPV1 on the activation of spinal cord glia in mouse models of pain. Exp Neurol 2009; 220: 383-90.

[95] Hu F, Sun WW, Zhao XT, et al. TRPV1 mediates cell death in rat synovial fibroblasts through calcium entry-dependent ROS production and mitochondrial depolarization. Biochem Biophys Res Commun 2008; 369: 989-93.

[96] Li X, Gibson G, Kim JS, et al. MicroRNA-146a is linked to painrelated pathophysiology of osteoarthritis. Gene 2011; 480: 34-41.

[97] Alawi K, Keeble J. The paradoxical role of the transient receptor potential vanilloid 1 receptor in inflammation. Pharmacol Ther 2010; 125: 181-95.

[98] Fernandes ES, Fernandes M, Keeble J. The functions of TRPA1 and TRPV1: moving away from sensory nerves. Br J Pharmacol 2012; 166: 510-21.

[99] Brain SD, Williams TJ, Tippins JR, et al. Calcitonin gene-related peptide is a potent vasodilator. Nature $1985 ; 313$ : 54-6.

[100] Brain SD, Grant AD. Vascular actions of calcitonin gene-related peptide and adrenomedullin. Physiol Rev 2004; 84: 903-34.

[101] Cambridge H, Brain SD. Calcitonin gene-related peptide increases blood flow and potentiates plasma protein extravasation in the rat knee joint. Br J Pharmacol 1992; 106: 746-50.

[102] Mapp PI, McWilliams DF, Turley MJ, et al. A role for the sensory neuropeptide calcitonin gene-related peptide in endothelial cell proliferation in vivo. Br J Pharmacol 2012; 166(4): 1261-71.

[103] Cady RJ, Glenn JR, Smith KM, et al. Calcitonin gene-related peptide promotes cellular changes in trigeminal neurons and glia implicated in peripheral and central sensitization. Mol Pain 2011; 7: 94.

[104] Moore EL, Salvatore CA. Targeting a family B GPCR/RAMP receptor complex: CGRP receptor antagonists and migraine. $\mathrm{Br} \mathrm{J}$ Pharmacol 2012; 166: 66-78.

[105] Davis JB, Gray J, Gunthorpe MJ, et al. Vanilloid receptor-1 is essential for inflammatory thermal hyperalgesia. Nature 2000; 405 : 183-7.

[106] Gunthorpe MJ, Szallasi A. Peripheral TRPV1 receptors as targets for drug development: new molecules and mechanisms. Curr Pharm Des 2008; 14: 32-41.
[107] Fernihough J, Gentry C, Bevan S, et al. Regulation of calcitonin gene-related peptide and TRPV1 in a rat model of osteoarthritis. Neurosci Lett 2005; 388: 75-80.

[108] Ferreira-Gomes J, Adães S, Sarkander J, et al. Phenotypic alterations of neurons that innervate osteoarthritic joints in rats. Arthritis Rheum 2010; 62: 3677-85.

[109] Puttfarcken PS, Han P, Joshi SK, et al. A-995662 [(R)-8-(4methyl-5-(4-(trifluoromethyl)phenyl)oxazol-2-ylamino)-1,2,3,4tetrahydronaphthalen-2-ol], a novel, selective TRPV1 receptor antagonist, reduces spinal release of glutamate and CGRP in a rat knee joint pain model. Pain 2010; 150: 319-26.

[110] Hoffmeister C, Trevisan G, Rossato MF, et al. Role of TRPV1 in nociception and edema induced by monosodium urate crystals in rats. Pain 2011; 152: 1777-88.

[111] Story GM, Peier AM, Reeve AJ, et al. ANKTM1, a TRP-like channel expressed in nociceptive neurons, is activated by cold temperatures. Cell 2003; 112: 819-29.

[112] Andersson DA, Gentry C, Moss S, et al. Transient receptor potential A1 is a sensory receptor for multiple products of oxidative stress. J Neurosci 2008; 28: 2485-94.

[113] Bessac BF, Sivula M, von Hehn CA, et al. TRPA1 is a major oxidant sensor in murine airway sensory neurons. J Clin Invest 2008; 118: 1899-910.

[114] Cruz-Orengo L, Dhaka A, Heuermann RJ, et al. Cutaneous nociception evoked by 15 -delta PGJ2 via activation of ion channel TRPA1. Mol Pain 2008; 4: 30 .

[115] Shan ZZ, Masuko-Hongo K, Dai SM, et al. A potential role of 15 deoxy-delta(12,14)-prostaglandin $\mathrm{J} 2$ for induction of human articular chondrocyte apoptosis in arthritis. J Biol Chem 2004; 279 : 37939-50.

[116] Lin TH, Tang $\mathrm{CH}, \mathrm{Wu} \mathrm{K}$, et al. 15-deoxy- $\Delta(12,14)$-prostaglandin$\mathrm{J} 2$ and ciglitazone inhibit TNF- $\alpha$-induced matrix metalloproteinase 13 production via the antagonism of NF- $\kappa \mathrm{B}$ activation in human synovial fibroblasts. J Cell Physiol 2011; 226: 3242-50.

[117] Chi PL, Chen YW, Hsiao LD, et al. HO-1 attenuates IL-1 $\beta$-induced cPLA(2) expression via a decrease in NADPH oxidase/ROS/AP-1 activation in human rheumatoid arthritis synovial fibroblasts. Arthritis Rheum 2012; 64(7): 2114-25.

[118] Xie L, Lin AS, Kundu K, et al. Quantitative imaging of cartilage and bone morphology, reactive oxygen species, and vascularization in a rodent model of osteoarthritis. Arthritis Rheum 2012; 64(7): 1899-908.

[119] Brain SD. TRPV1 and TRPA1 channels in inflammatory pain: elucidating mechanisms. Ann N Y Acad Sci 2011; 1245: 36-7.

[120] Eid SR. Therapeutic targeting of TRP channels--the TR(i)P to pain relief. Curr Top Med Chem 2011; 11: 2118-30.

[121] Andrade EL, Meotti FC, Calixto JB. TRPA1 antagonists as potential analgesic drugs. Pharmacol Ther 2012; 133: 189-204.

[122] Earley S, Gonzales AL, Crnich R. Endothelium-dependent cerebral artery dilation mediated by TRPA1 and $\mathrm{Ca} 2+$-Activated $\mathrm{K}+$ channels. Circ Res 2009; 104: 987-94.

[123] Obata K, Katsura H, Mizushima T, et al. TRPA1 induced in sensory neurons contributes to cold hyperalgesia after inflammation and nerve injury. J Clin Invest 2005; 115: 2393-401.

[124] Gorin AA, Smyth JM, Weisberg JN, et al. Rheumatoid arthritis patients show weather sensitivity in daily life, but the relationship is not clinically significant. Pain 1999; 81: 173-7.

[125] Lee YC, Lu B, Bathon JM, et al. Pain sensitivity and pain reactivity in osteoarthritis. Arthritis Care Res (Hoboken) 2011; 63: 320-7.

[126] Kwan KY, Corey DP. Burning cold: involvement of TRPA1 in noxious cold sensation. J Gen Physiol 2009; 133: 251-6.

[127] Strübing C, Krapivinsky G, Krapivinsky L, et al. TRPC1 and TRPC 5 form a novel cation channel in mammalian brain. Neuron 2001; 29: 645-55.

[128] Suzuki M, Mizuno A, Kodaira K, et al. Impaired pressure sensation in mice lacking TRPV4. J Biol Chem 2003; 278:22664-8.

[129] Xu SZ, Sukumar P, Zeng F, et al. TRPC channel activation by extracellular thioredoxin. Nature 2008; 451: 69-72.

[130] Itoh $\mathrm{Y}$, Hatano $\mathrm{N}$, Hayashi $\mathrm{H}$, et al. An environmental sensor, TRPV4 is a novel regulator of intracellular $\mathrm{Ca}^{2+}$ in human synoviocytes. Am J Physiol Cell Physiol 2009; 297: C1082-90.

[131] Sossey-Alaoui K, Lyon JA, Jones L, et al. Molecular cloning and characterization of TRPC5 (HTRP5), the human homologue of a mouse brain receptor-activated capacitative $\mathrm{Ca}^{2+}$ entry channel. Genomics 1999; 60: 330-40. 
[132] Zimmermann K, Lennerz JK, Hein A, et al. Transient receptor potential cation channel, subfamily $\mathrm{C}$, member 5 (TRPC5) is a cold-transducer in the peripheral nervous system. Proc Natl Acad Sci U S A 2011; 108: 18114-9.

[133] Tsuji G, Koshiba M, Nakamura H, et al. Thioredoxin protects against joint destruction in a murine arthritis model. Free Radic Biol Med 2006; 40: 1721-31.

[134] Nakamura H, Hoshino Y, Okuyama H, et al. Thioredoxin 1 delivery as new therapeutics. Adv Drug Deliv Rev 2009; 61: 303-9.

[135] Yoshida T, Inoue R, Morii T, et al. Nitric oxide activates TRP channels by cysteine S-nitrosylation. Nat Chem Biol 1999; 2: 596607.

[136] Lee H, Iida T, Mizuno A, et al. Altered thermal selection behavior in mice lacking transient receptor potential vanilloid 4. J Neurosci 2005; 25: 1304-10.

[137] Clark AL, Votta BJ, Kumar S, et al. Chondroprotective role of the osmotically sensitive ion channel transient receptor potential vanilloid 4: age- and sex-dependent progression of osteoarthritis in Trpv4-deficient mice. Arthritis Rheum 2010; 62: 2973-83.

[138] Massullo P, Sumoza-Toledo A, Bhagat H, et al. TRPM channels, calcium and redox sensors during innate immune responses. Semin Cell Dev Biol 2006; 17: 654-66.
[139] McMeekin SR, Dransfield I, Rossi AG, et al. E-selectin permits communication between PAF receptors and TRPC channels in human neutrophils. Blood 2006; 107: 4938-45.

[140] Gavenis K, Schumacher C, Schneider U, et al. Expression of ion channels of the TRP family in articular chondrocytes from osteoarthritic patients: changes between native and in vitro propagated chondrocytes. Mol Cell Biochem 2009; 321: 135-43.

[141] Ciurtin C, Majeed Y, Naylor J, et al. TRPM3 channel stimulated by pregnenolone sulphate in synovial fibroblasts and negatively coupled to hyaluronan. BMC Musculoskelet Disord 2010; 11: 111.

[142] Finney-Hayward TK, Popa MO, Bahra P, et al. Expression of transient receptor potential C6 channels in human lung macrophages. Am J Respir Cell Mol Biol 2010; 43: 296-304.

[143] Wolfe F, Mitchell DM, Sibley JT, et al. The mortality of rheumatoid arthritis. Arthritis Rheum 1994; 37: 481-94.

[144] Witter J, Dionne RA. What can chronic arthritis pain teach about developing new analgesic drugs? Arthritis Res Ther 2004; 6: 27981 .

[145] Home D, Carr M. Rheumatoid arthritis: the role of early intervention and self-management. Br J Community Nurs 2009; 14 : $432-6$.

(C) Fernandes et al.; Licensee Bentham Open.

This is an open access article licensed under the terms of the Creative Commons Attribution Non-Commercial License (http://creativecommons.org/licenses/by-nc/3.0/) which permits unrestricted, non-commercial use, distribution and reproduction in any medium, provided the work is properly cited. 